\title{
Empirical transverse charge densities in the deuteron
}

\author{
C.E. Carlson ${ }^{1}$ and M. Vanderhaeghen ${ }^{1,2, a}$ \\ 1 Physics Department, College of William and Mary, Williamsburg, VA 23187, USA \\ 2 Institut für Kernphysik, Johannes Gutenberg-Universität, D-55099 Mainz, Germany
}

Received: 21 February 2009

Published online: 21 May 2009 - (c) Società Italiana di Fisica / Springer-Verlag 2009

Communicated by M. Anselmino

\begin{abstract}
Using the recent empirical information on the deuteron electromagnetic form factors we map out the transverse charge density in the deuteron as viewed from a light front moving towards the deuteron. The charge densities for a transversely polarized deuteron are characterized by monopole, dipole and quadrupole patterns.
\end{abstract}

PACS. 13.40.Gp Electromagnetic form factors - 21.10.Ft Charge distribution - 21.45.Bc Two-nucleon system

Electromagnetic form factors (FFs) of the deuteron have received a lot of attention in recent years; for recent reviews see, e.g., refs. [1,2]. Based on the large amount of precise deuteron form factor data, it is therefore of interest to exhibit the spatial information on the quark charge distributions in the deuteron. In this letter, we develop the general formalism to extract charge densities for a spin-1 particle and apply it to the case of the deuteron.

In the following we will consider the electromagnetic deuteron elastic FFs when viewed from a light front moving towards the deuteron. Equivalently, this corresponds with a frame where the deuterons have a large momentum component along the $z$-axis chosen along the direction of $P=\left(p+p^{\prime}\right) / 2$, where $p\left(p^{\prime}\right)$ are the initial (final) deuteron four-momenta. We indicate the deuteron light front + component by $P^{+}$(defining $a^{ \pm} \equiv a^{0} \pm a^{3}$ ). We furthermore choose a light front frame where the virtual photon four-momentum $q$ has $q^{+}=0$, and has a transverse component (lying in the $x y$-plane) indicated by the transverse vector $\boldsymbol{q}_{\perp}$, satisfying $q^{2}=-\boldsymbol{q}_{\perp}{ }^{2} \equiv-Q^{2}$. In such a light front frame, the virtual photon only couples to forward-moving partons and the + component of the electromagnetic current $J^{+}$has the interpretation of the quark charge density operator. It is given by

$$
J^{+}(0)=+2 / 3 \bar{u}(0) \gamma^{+} u(0)-1 / 3 \bar{d}(0) \gamma^{+}(0) d(0),
$$

considering only $u$ and $d$ quarks. Each term in the expression is a positive operator since $\bar{q} \gamma^{+} q \propto\left|\gamma^{+} q\right|^{2}$.

In the following, we will use empirical information on deuteron elastic FFs to study the deuteron quark charge

\footnotetext{
a e-mail: marcvdh@kph.uni-mainz.de
}

densities in the transverse plane. It is customary to denote the three deuteron elastic e.m. FFs by $G_{C}, G_{M}$, and $G_{Q}$, corresponding to the Coulomb monopole $\left(G_{C}\right)$, magnetic dipole $\left(G_{M}\right)$, and Coulomb quadrupole $\left(G_{Q}\right)$ FFs, respectively. Similar relations between nucleon densities and FFs can be found in [3-6].

We start by expressing the matrix elements of the $J^{+}(0)$ operator between deuteron states as

$$
\begin{aligned}
\left\langle P^{+}, \frac{\boldsymbol{q}_{\perp}}{2}, \lambda^{\prime}\left|J^{+}(0)\right| P^{+},-\frac{\boldsymbol{q}_{\perp}}{2}, \lambda\right\rangle= & \left(2 P^{+}\right) e^{i\left(\lambda-\lambda^{\prime}\right) \phi_{q}} \\
& \times G_{\lambda^{\prime} \lambda}^{+}\left(Q^{2}\right),
\end{aligned}
$$

where $\lambda= \pm 1,0\left(\lambda^{\prime}= \pm 1,0\right)$ denotes the initial (final) deuteron light front helicity, and where $\boldsymbol{q}_{\perp}=Q\left(\cos \phi_{q} \hat{e}_{x}+\right.$ $\sin \phi_{q} \hat{e}_{y}$ ). Furthermore in eq. (2), the helicity form factors $G_{\lambda^{\prime} \lambda}^{+}$are real (due to time reversal invariance) and depend only on $Q^{2}$.

We can then define transverse charge densities for a deuteron in helicity states of $\lambda= \pm 1$ or $\lambda=0$ as,

$$
\begin{aligned}
\rho_{\lambda}^{d}(b) & \equiv \int \frac{\mathrm{d}^{2} \boldsymbol{q}_{\perp}}{(2 \pi)^{2}} e^{-i \boldsymbol{q}_{\perp} \cdot \boldsymbol{b}} \frac{1}{2 P^{+}}\left\langle P^{+}, \frac{\boldsymbol{q}_{\perp}}{2}, \lambda\left|J^{+}\right| P^{+}, \frac{-\boldsymbol{q}_{\perp}}{2}, \lambda\right\rangle \\
& =\int_{0}^{\infty} \frac{\mathrm{d} Q}{2 \pi} Q J_{0}(b Q) G_{\lambda \lambda}^{+}\left(Q^{2}\right),
\end{aligned}
$$

where $\boldsymbol{b} \equiv b\left(\cos \phi_{b} \hat{e}_{x}+\sin \phi_{b} \hat{e}_{y}\right)$ denotes the position in the $x y$-plane from the transverse c.m. of the deuteron. The two independent helicity-conserving FFs $G_{11}^{+}$and $G_{00}^{+}$can 
be expressed in terms of $G_{C, M, Q}$ as,

$$
\begin{aligned}
G_{11}^{+} & =\frac{1}{1+\eta}\left\{G_{C}+\eta G_{M}+\frac{\eta}{3} G_{Q}\right\}, \\
G_{00}^{+} & =\frac{1}{1+\eta}\left\{(1-\eta) G_{C}+2 \eta G_{M}-\frac{2 \eta}{3}(1+2 \eta) G_{Q}\right\},
\end{aligned}
$$

with $\eta \equiv Q^{2} /\left(4 M_{d}^{2}\right)$, and $M_{d}$ is the deuteron mass.

The definitions and normalizations of $G_{C}, G_{M}$, and $G_{Q}$ are the customary ones obtained from the matrix elements of the electromagnetic current [7],

$$
\begin{aligned}
& \left\langle p_{2}, \lambda_{2}\left|J^{\mu}\right| p_{1}, \lambda_{1}\right\rangle=-\left(\varepsilon_{2}^{*} \cdot \varepsilon_{1}\right) 2 P^{\mu} G_{1}\left(Q^{2}\right) \\
& -\left(\varepsilon_{1}^{\mu} \varepsilon_{2}^{*} \cdot q-\varepsilon_{2}^{\mu *} \varepsilon_{1} \cdot q\right) G_{M}\left(Q^{2}\right)+q \cdot \varepsilon_{1} q \cdot \varepsilon_{2}^{*} \frac{P^{\mu}}{M_{d}^{2}} G_{3}\left(Q^{2}\right),
\end{aligned}
$$

with $\varepsilon_{1,2}$ the polarization vectors of the initial and final deuteron. The charge and quadrupole FFs follow from,

$$
\begin{aligned}
G_{C} & =G_{1}+\frac{2}{3} \eta G_{Q}, \\
G_{Q} & =G_{1}+(1+\eta) G_{3}-G_{M},
\end{aligned}
$$

with normalizations $G_{C}(0)=1, G_{M}(0)=\mu_{d}$ (magnetic moment in units $e /\left(2 M_{d}\right)$ ), and $G_{Q}(0)=Q_{d}$ (quadrupole moment in units $\left.e / M_{d}^{2}\right)$.

For numerical evaluation, we use the parameterization of the deuteron form factor data given as fit II by Abbott et al. [8]. This parameterization is based on forms suggested in [9], which read

$$
\begin{aligned}
G_{C} & =\frac{G^{2}\left(\frac{Q^{2}}{4}\right)}{2 \eta+1}\left[\left(1-\frac{2}{3} \eta\right) g_{0}+\frac{8}{3} \sqrt{2 \eta} g_{1}+\frac{2}{3}(2 \eta-1) g_{2}\right] \\
G_{M} & =\frac{G^{2}\left(\frac{Q^{2}}{4}\right)}{2 \eta+1}\left[2 g_{0}+\frac{2(2 \eta-1)}{\sqrt{2 \eta}} g_{1}-2 g_{2}\right] \\
G_{Q} & =\frac{G^{2}\left(\frac{Q^{2}}{4}\right)}{2 \eta+1}\left[-g_{0}+\sqrt{\frac{2}{\eta}} g_{1}-\frac{\eta+1}{\eta} g_{2}\right]
\end{aligned}
$$

where the dipole form factor $G\left(Q^{2}\right)=\left(1+Q^{2} / \delta^{2}\right)^{-2}$ has a (non-standard) mass parameter $\delta=898.52 \mathrm{MeV}$. The reduced amplitudes are

$g_{0}=\sum_{i=1}^{4} \frac{a_{i}}{\alpha_{i}^{2}+Q^{2}}, \quad g_{1}=\sum_{i=1}^{4} \frac{Q b_{i}}{\beta_{i}^{2}+Q^{2}}, \quad g_{2}=\sum_{i=1}^{4} \frac{Q^{2} c_{i}}{\gamma_{i}^{2}+Q^{2}}$,

and the parameters from [8] are given in table 1 .

Figure 1 shows the transverse charge densities for definite helicity (longitudinally polarized) deuterons. Recall that the transverse charge density is the charge density projected onto a plane perpendicular to the line of sight, which also defines the longitudinal direction. The charge density for the $\lambda=1$ state, in the upper panel, is smooth and peaks in the center. The $\lambda=0$ state, in the middle panel, features a dip in the center, which can also be seen
Table 1. Parameters for the deuteron form factor fit II of ref. [8].

\begin{tabular}{c|c|c|c|c}
\hline & $i=1$ & $i=2$ & $i=3$ & $i=4$ \\
\hline$a_{i}\left(\mathrm{fm}^{-2}\right)$ & 1.57057 & 12.23792 & -42.04576 & 27.92014 \\
$b_{i}\left(\mathrm{fm}^{-1}\right)$ & 0.07043 & 0.14443 & -0.27343 & 0.05856 \\
$c_{i}$ & -0.16577 & 0.27557 & -0.05382 & -0.05598 \\
$\alpha_{i}^{2}\left(\mathrm{fm}^{-2}\right)$ & 1.52501 & 8.75139 & 15.97777 & 23.20415 \\
$\beta_{i}^{2}\left(\mathrm{fm}^{-2}\right)$ & 43.67795 & 30.05435 & 16.43075 & 2.80716 \\
$\gamma_{i}^{2}\left(\mathrm{fm}^{-2}\right)$ & 1.87055 & 14.95683 & 28.04312 & 41.12940 \\
\hline
\end{tabular}
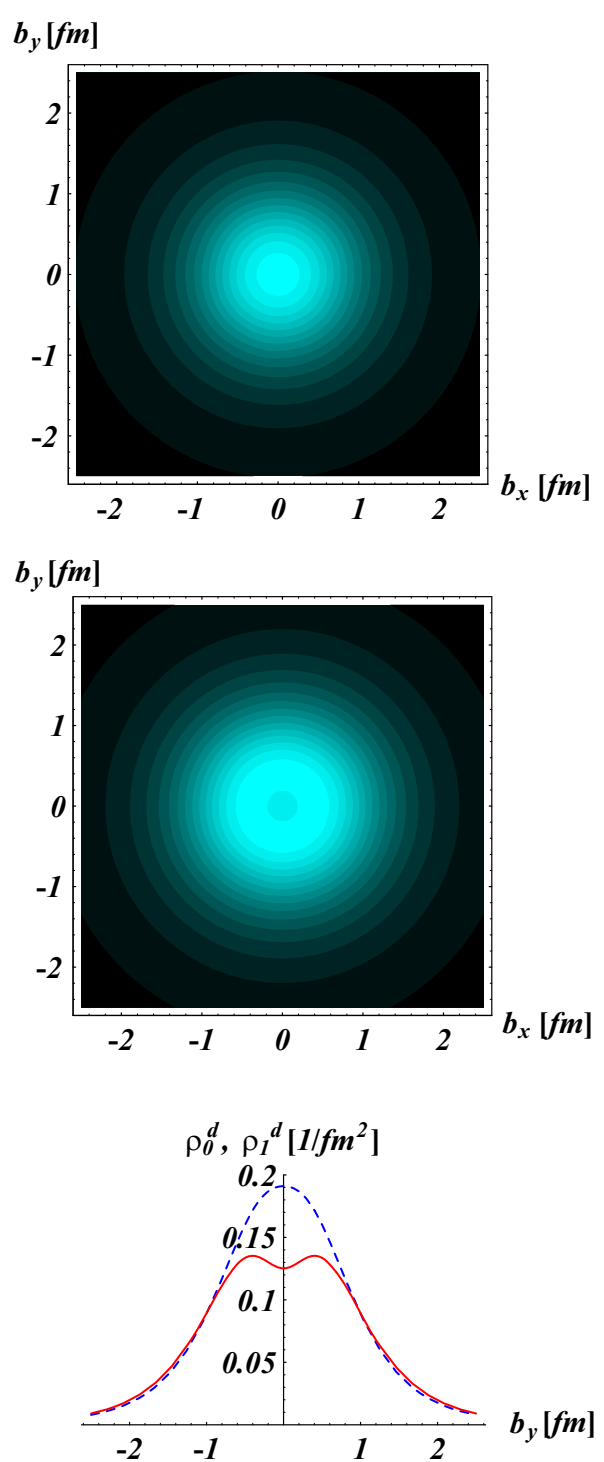

Fig. 1. Quark transverse charge densities in the deuteron. Upper panel: $\rho_{1}^{d}$. Middle panel: $\rho_{0}^{d}$. The lightest (darkest) regions correspond with largest (smallest) densities. Lower panel: the density along the $y$-axis for $\rho_{1}^{d}$ (dashed curve) and $\rho_{0}^{d}$ (solid curve). For the deuteron FFs, we use the empirical parameterization II of ref. [8], based on ref. [9].

in the lower panel, where the density along the $y$-axis is plotted for both states. 
This dip - found directly from the data - reflects the low charge density or "hole" in the center-of-thedeuteron wave function. For the helicity-0 state, calculated $S$ - and $D$-state wave functions produce toroidal equidensity surfaces in the central regions, with the axis along the $z$-direction [10]. Hence looking along the $z$-axis for this state, one can see the hole in the center, albeit the hole be partly filled in because one must look though the outer layers of the deuteron which are mostly $S$-state and spherically symmetric. We emphasize that the dip seen in our figure is based only on observation (codified in the form factor fits) and a light front interpretation of the data. The result is consistent with but does not use calculations based on models of nucleon-nucleon interactions. Further, the light front viewpoint gives a two-dimensional charge density that is relativistically correct, unlike charge densities obtained from Fourier transforming FFs in an equal time formalism. The light front viewpoint also gives a neutron central charge density which is negative [4], because it involves the Fourier transform of the Dirac FF $F_{1}$ instead of the Sachs FF $G_{E}$. More physically, the negative core may be understood [11] as quarks at small impact parameter correlating with high momenta in all directions, noting that high longitudinal momentum quarks in a neutron are dominantly down quarks.

We next consider the charge densities for a transversely polarized deuteron, denoting the transverse polarization direction by $\boldsymbol{S}_{\perp}=\cos \phi_{S} \hat{e}_{x}+\sin \phi_{S} \hat{e}_{y}$. The transverse charge densities can be defined as

$$
\begin{aligned}
\rho_{T s_{\perp}}^{d}(\boldsymbol{b}) \equiv & \int \frac{\mathrm{d}^{2} \boldsymbol{q}_{\perp}}{(2 \pi)^{2}} e^{-i \boldsymbol{q}_{\perp} \cdot \boldsymbol{b}} \frac{1}{2 P^{+}} \\
& \times\left\langle P^{+}, \frac{\boldsymbol{q}_{\perp}}{2}, s_{\perp}\left|J^{+}\right| P^{+}, \frac{-\boldsymbol{q}_{\perp}}{2}, s_{\perp}\right\rangle,
\end{aligned}
$$

where $s_{\perp}$ is the deuteron spin projection along the direction of $\boldsymbol{S}_{\perp}$. The transverse spin state can be expressed in terms of the light front helicity spinor states as

$$
\begin{aligned}
\left|s_{\perp}= \pm 1\right\rangle= & \frac{1}{2}\left(|\lambda=+1\rangle \pm \sqrt{2} e^{i \phi_{S}}|\lambda=0\rangle\right. \\
& \left.+e^{2 i \phi_{S}}|\lambda=-1\rangle\right) \\
\left|s_{\perp}=0\right\rangle= & \frac{1}{\sqrt{2}}\left(|\lambda=+1\rangle-e^{2 i \phi_{S}}|\lambda=-1\rangle\right) .
\end{aligned}
$$

By working out the Fourier transform in eq. (9) for the two cases where $s_{\perp}=+1$ and $s_{\perp}=0$, one obtains

$$
\begin{aligned}
\rho_{T 1}^{d}(\boldsymbol{b})= & \int_{0}^{\infty} \frac{\mathrm{d} Q}{2 \pi} Q\left\{J_{0}(b Q) \frac{1}{2}\left(G_{11}^{+}+G_{00}^{+}\right)\right. \\
& +\sin \left(\phi_{b}-\phi_{S}\right) J_{1}(b Q) \sqrt{2} G_{01}^{+} \\
& \left.-\cos 2\left(\phi_{b}-\phi_{S}\right) J_{2}(b Q) \frac{1}{2} G_{-1+1}^{+}\right\}, \\
\rho_{T 0}^{d}(\boldsymbol{b})= & \int_{0}^{\infty} \frac{\mathrm{d} Q}{2 \pi} Q\left\{J_{0}(b Q) G_{11}^{+}\right. \\
& \left.+\cos 2\left(\phi_{b}-\phi_{S}\right) J_{2}(b Q) G_{-1+1}^{+}\right\} .
\end{aligned}
$$

One notices from eqs. (11), (12) that the transverse charge density $\rho_{T 1}^{d}$ contains monopole, dipole and quadrupole field patterns, whereas $\rho_{T 0}^{d}$ only contains monopole and quadrupole field patterns. The deuteron helicity FF with one unit of helicity flip, which governs the dipole field pattern in $\rho_{T 1}^{d}$, can be expressed in terms of $G_{C, M, Q}$ as

$$
G_{01}^{+}=-\frac{\sqrt{2 \eta}}{1+\eta}\left\{G_{C}-\frac{1}{2}(1-\eta) G_{M}+\frac{\eta}{3} G_{Q}\right\},
$$

whereas the deuteron helicity FF with two units of helicity flip, giving the quadrupole field patterns, is,

$$
G_{-1+1}^{+}=\frac{\eta}{1+\eta}\left\{G_{C}-G_{M}-\left(1+\frac{2 \eta}{3}\right) G_{Q}\right\} .
$$

The four-deuteron helicity FFs given here are not independent. The angular condition [12] relating them is,

$$
(2 \eta+1) G_{11}^{+}+2 \sqrt{2 \eta} G_{01}^{+}+G_{-1+1}^{+}-G_{00}^{+}=0 .
$$

Magnetic dipole moments in a rest frame appear, because of relativity, as electric dipole moments in a fastmoving frame [13]. The electric dipole moment (EDM) corresponding to the transverse charge densities $\rho_{T s_{\perp}}^{d}$ is

$$
\boldsymbol{d}_{s_{\perp}}^{d} \equiv e \int \mathrm{d}^{2} \boldsymbol{b} \boldsymbol{b} \rho_{T s_{\perp}}^{d}(\boldsymbol{b}) .
$$

Equation (12) gives $\boldsymbol{d}_{0}^{d}=0$, whereas eq. (11) yields

$$
\boldsymbol{d}_{1}^{d}=-\left(\boldsymbol{S}_{\perp} \times \hat{e}_{z}\right)\left\{G_{M}(0)-2\right\}\left(\frac{e}{2 M_{d}}\right) .
$$

Expressing the spin-1 magnetic moment in terms of the $g$-factor, i.e. $G_{M}(0)=g$, one sees that the induced EDM $\boldsymbol{d}_{1}^{d}$ is proportional to $g-2$. The same result was found for the case of a spin- $1 / 2$ particle [5]. One thus observes that for a particle without internal structure (corresponding with $g=2[14]$ ), there is no induced EDM.

The electric quadrupole moment corresponding with the transverse charge densities $\rho_{T s_{\perp}}^{d}$ is, for $\boldsymbol{S}_{\perp}=\hat{e}_{x}$,

$$
Q_{s_{\perp}}^{d} \equiv e \int \mathrm{d}^{2} \boldsymbol{b}\left(b_{x}^{2}-b_{y}^{2}\right) \rho_{T s_{\perp}}^{d}(\boldsymbol{b}) .
$$

From eqs. (11), (12) one obtains,

$$
\begin{aligned}
Q_{1}^{d} & =-\frac{1}{2} Q_{0}^{d} \\
& =\frac{1}{2}\left\{\left[G_{M}(0)-2\right]+\left[G_{Q}(0)+1\right]\right\}\left(\frac{e}{M_{d}^{2}}\right) .
\end{aligned}
$$

For a spin-1 particle without internal structure, exemplified by $W$ and $Z$ gauge bosons in standard electroweak theory, it is required that at tree level $G_{M}(0)=2$ and $G_{Q}(0)=-1$; these values also allow satisfying the Gerasimov-Drell-Hearn sum rule to lowest order in perturbation theory $[15,16]$. For elementary particles, any deviations from these values would indicate new (beyond 

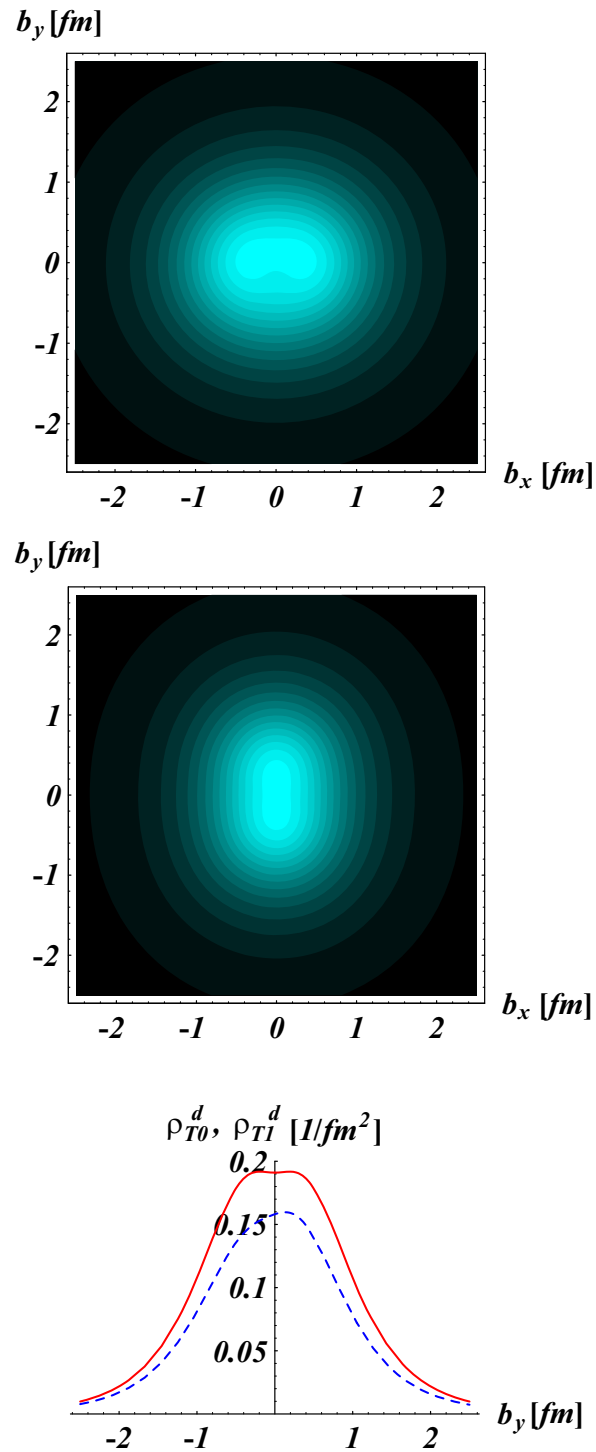

Fig. 2. Quark transverse charge densities for a deuteron which is polarized along the positive $x$-axis. Upper panel: $\rho_{T 1}^{d}$. Middle panel: $\rho_{T 0}^{d}$. The light (dark) regions correspond with largest (smallest) densities. The lower panel compares the density along the $y$-axis for $\rho_{T 1}^{d}$ (dashed curve) and $\rho_{T \text { 0 }}^{d}$ (solid curve). For the deuteron e.m. FFs, we use the empirical parameterization II of ref. [8], based on ref. [9].

standard model) physics. (Current data from $p \bar{p}$ collisions, up to $\sqrt{s}=1.96 \mathrm{TeV}$, are compatible with zero anomalous $W W \gamma$ and $Z Z \gamma$ couplings [17].) For composite particles, it is the deviations from these benchmark values that indicate deformations of the states.

It is thus interesting to observe from eq. (19) that $Q_{s}^{d}$ is only sensitive to the anomalous parts of the spin-1 magnetic dipole and electric quadrupole moments, and vanishes for a particle without internal structure. The deuteron magnetic dipole moment $G_{M}(0)$ is 1.71 [18], close to a spin-1 particle's natural value. However, in contrast to the $W$ and $Z$ gauge bosons, the deuteron has a large anomalous quadrupole moment. Its measured value is $G_{Q}(0)=25.84(3)$ [19], highlighting the prominent role of the pion exchange potential.

Pictorial results for the transverse charge density with transverse deuteron polarization are shown in fig. 2 . The upper panel shows the charge distribution for the state with projection- 1 in the $x$-direction. One sees the large effects of the quadrupole term together with a small overall shift of the charge distribution along the $y$-axis. The latter contrasts to the large shift of the charge in the neutron case [5], where the anomalous moment is far from its pointlike value. As noted $[13,20]$, an object with a magnetic dipole moment when stationary exhibits an electric dipole moment when seen by a moving observer, proportional to the vector product $\boldsymbol{v} \times$ (magnetic moment). The middle panel shows the charge density for a state with projection0 in the $x$-direction. One notices that the quadrupole term stretches the charge along the $y$-axis but does not cause any shift of the charge center. The two cases are compared in the lower panel, which plots the density along the $y$-axis.

In summary, we used recent empirical information on the deuteron e.m. FFs to map out the transverse charge densities in longitudinally and in transversely polarized deuterons. Notably, one sees a dip in the center-of-thecharge distribution for helicity-zero deuterons. This is in harmony with nuclear force model calculations, which for the zero helicity case predict toroidal equidensity surfaces for higher densities, with the axis along the quantization direction. Transversely polarized deuterons show dipole and quadrupole structure in the charge distributions. Their electric dipole and quadrupole moments only depend on the spin-1 particle's anomalous magnetic dipole moment and its anomalous electric quadrupole moment, arising from its internal structure.

The work of C.E.C. is supported by the NSF under grant PHY0555600. The work of M.V. is supported in part by DOE grant DE-FG02-04ER41302.

\section{References}

1. M. Garcon, J.W. Van Orden, Adv. Nucl. Phys. 26, 293 (2001).

2. R.A. Gilman, F. Gross, J. Phys. G 28, R37 (2002).

3. B. Pasquini, S. Boffi, Phys. Lett. B 653, 23 (2007).

4. G.A. Miller, Phys. Rev. Lett. 99, 112001 (2007).

5. C.E. Carlson, M. Vanderhaeghen, Phys. Rev. Lett. 100, 032004 (2008).

6. G.A. Miller, E. Piasetzky, G. Ron, Phys. Rev. Lett. 101, 082002 (2008).

7. R.G. Arnold, C.E. Carlson, F. Gross, Phys. Rev. C 21, 1426 (1980).

8. JLAB t20 Collaboration (D. Abbott et al.), Eur. Phys. J. A 7, 421 (2000).

9. A.P. Kobushkin, A.I. Syamtomov, Phys. At. Nucl. 58, 1477 (1995) (Yad. Fiz. 58, 1565 (1995)).

10. J.L. Forest, V.R. Pandharipande, S.C. Pieper, R.B. Wiringa, R. Schiavilla, A. Arriaga, Phys. Rev. C 54, 646 (1996). 
11. G.A. Miller, J. Arrington, Phys. Rev. C 78, 032201 (2008).

12. C.E. Carlson, C.R. Ji, Phys. Rev. D 67, 116002 (2003).

13. A. Einstein, J. Laub, Ann. Phys. (Leipzig) 331, 532 (1908).

14. S. Ferrara, M. Porrati, V.L. Telegdi, Phys. Rev. D 46, 3529 (1992).

15. K.J. Kim, Y.S. Tsai, Phys. Rev. D 7, 3710 (1973).

16. S.J. Brodsky, J.R. Hiller, Phys. Rev. D 46, 2141 (1992).
17. D0 Collaboration (V.M. Abazov et al.), Phys. Rev. Lett. 100, 241805 (2008); Phys. Lett. B 653, 378 (2007).

18. P.J. Mohr, B.N. Taylor, D.B. Newell, Rev. Mod. Phys. 80, 633 (2008) (CODATA 2006).

19. T.E.O. Ericson, M. Rosa-Clot, Nucl. Phys. A 405, 497 (1983).

20. R.V. Krotkov, G.N. Pellegrini, N.C. Ford, A.R. Swift, Am. J. Phys. 67, 493 (1999). 